\title{
Blood Lactate, Body Temperature and Heart Rate During Sub- maximal Exercise in Dogs with Brachycephalic Obstructive Airway Syndrome: A Preliminary Study
}

\author{
Žgank $\check{Z}^{1}$, Nemec Svete $A^{1}$, Erjavec $\mathbf{V}^{1 *}$ \\ 1. University of Ljubljana, Veterinary Faculty, Small Animal Clinic, Ljubljana, Slovenia \\ * Correspondence: Vladimira Erjavec: vladimira.erjavec@vf.uni-lj.si
}

Citation: Žgank Ž, Nemec Svete A, Erjavec V. Blood lactate, body temperature and heart rate during submaximal exercise in dogs with brachycephalic obstructive airway syndrome: a preliminary study. Proceedings of Socratic Lectures. 2021; 6: 8-13.

https://doi.org/10.55295/PSL.2021.D. 002

Publisher's Note: UL ZF stays neutral with regard to jurisdictional claims in published maps and institutional affiliations.

\begin{abstract}
The aim of the present preliminary study was to investigate how submaximal exercise affects blood lactate (BL) concentrations, body temperature (BT) and heart rate (HR) in dogs with brachycephalic obstructive airway syndrome (BOAS). Seven dogs with BOAS grade $2 / 3$ and $3 / 3$ were subjected to submaximal exercise on a treadmill. Each dog was subjected to training session that began with a 5 -minute walk at a speed of $2.5 \mathrm{~km} / \mathrm{h}$ and an incline of $0 \%$ and continued with a 5 -minute walk at a speed of $2.5 \mathrm{~km} / \mathrm{h}$ and at an incline of $5 \%$. Heart rate, BT, and BL concentrations were measured before the start (T1), every 5 minutes during the test (T2, T3) and after 15 and 30 minutes of rest (T4, T5). Blood lactate concentrations at T3 and T5 were significantly lower $(\mathrm{P}<0.05)$ than concentrations at T1. Heart rate and BT values at T2 and T3 were significantly higher $(\mathrm{P}<0.05)$ than values at T1. Submaximal exercise resulted in a significant decrease in BL concentrations and a significant increase in BT and HR in dogs with BOAS. The results of this preliminary study can give us additional information about the severity of BOAS; however, further studies are needed to gain better insight into the physiological response of BOAS patients of individual breeds to submaximal exercise testing.
\end{abstract}

Keywords: Brachycephalic obstructive airway syndrome; Treadmill; Brachycephalic dogs; Blood lactate; Submaximal exercise test
Copyright: (C) 2021 by the authors.

Submitted for possible open access

publication under the terms and conditions of the Creative Commons Attribution (CC BY) license

(https://creativecommons.org/licenses/b $\mathrm{y} / 4.0 /)$. 


\section{Introduction}

The popularity of brachycephalic dogs has increased in recent years. This may be due to their distinct physical facial features, which resemble the facial features of a human baby and elicit the same positive emotions in adults. Breeds such as French and English bulldogs, pugs, and Boston terriers are examples of extremely brachycephalic breeds. They have a typically shaped head with a very short muzzle and a round, short and broad head (Ekenstedt et al., 2020). The soft tissues of the upper airway have not reduced in proportion to the skull length and these deformities lead to upper airway obstruction (Dupré and Heidenreich, 2016). These upper airway abnormalities cause a variety of clinical signs called brachycephalic obstructive airway syndrome (BOAS) (Lodato and Hedlund, 2012). BOAS is a progressive disease. Dogs with BOAS may present with clinical signs such as stertor, stridor, snoring, coughing, increased respiratory effort, exercise intolerance, hyperthermia, and collapse. These problems may be exacerbated in the presence of stress, physical activity, and high ambient temperatures (Meola et al., 2013).

In dogs with cardiac, respiratory, or neuromuscular disease, exercise tests (ET) is used to assess the severity of the disease. In dogs with BOAS, ET can help identify clinically relevant airway obstruction when clinical signs are mild or dynamic (Riggs et al., 2019). In sports medicine blood lactate (BL) is one of the most important biochemical parameters measured during exercise. In both human and animal athletes, such as horses and dogs, BL is used to determine the level of fitness and effort (Alves et al., 2020). It is a metabolite of the anaerobic glycolytic pathways (Rovira et al., 2018). It is produced continuously under anaerobic conditions and has at least three functions: a major energy source, the major gluconeogenic precursor, and a signalling molecule (Brooks, 2018). In dogs, physical activity leads to higher heath and therefore higher cardiovascular and thermoregulatory demand (Robbins et al., 2017). Measurement of HR and BT during exercise is useful for monitoring exercise intensity and may also be useful for detecting subclinical disease (Rovira et al., 2018). Monitoring HR during exercise is also useful for determining cardiac output and monitoring workload. Monitoring HR during rest periods can help determine physical fitness and metabolic status (Alves et al., 2020).

The aim of this study was to determine how submaximal exercise affects the levels of $\mathrm{BL}, \mathrm{HR}$, and $\mathrm{BT}$ in dogs with BOAS.

\section{Materials and Methods}

\subsection{Animals}

Seven client-owned brachycephalic dogs, prospectively recruited as BOAS patients were included in the study. The dogs were graded with BOAS grade $2 / 3(n=2)$ and grade $3 / 3(n=5)$. BOAS grade was based on clinical signs of upper airway obstruction and anatomical abnormalities (Erjavec et al., 2021).

Formal written consent of the owner was obtained before the dogs participated in the study. All procedures complied with the relevant Slovenian governmental regulations (Animal Protection Act UL RS, 43/2007).

\subsection{Exercise test and measurements of $B L$ concentration, $H R$, and $B T$}

The exercise test was performed in the Laboratory of physiology, Faculty of sports, University of Ljubljana in a controlled climatic environment. The test was performed in two stages. The first stage was a 5-minute walk on a treadmill at a speed of $2.5 \mathrm{~km} / \mathrm{h}$ and a $0 \%$ incline. The second stage was a 5 -minute walk at a speed of $2.5 \mathrm{~km} / \mathrm{h}$ at an inclination of $5 \%$. There was a 1-minute break between both stages for measurements. After the exercise the dogs had 30 minutes of recovery time.

Heart rate, BT and BL concentrations were measured before the start (T1), after the first 5 minutes of exercise (T2), after 10 minutes (T3) of exercise, and after 15 minutes (T4) and 30 minutes of recovery (T5). Blood samples for measurement of BL concentrations were collected from the cephalic vein. Blood lactate concentration was measured immediately after blood was drawn using a portable device (Accutrend Plus, Roche Diagnostics 
$\mathrm{GmbH}$, Mannheim, Germany). Heart rate was measured by palpation of the femoral artery and counted for 15 seconds and then multiplied by four. Body temperature was measured rectally with digital thermometer (Microlife, Microlife AG, Widnau, Switzerland).

\subsection{Statistical analysis}

Data was analysed with commercial software (IBM SPSS 25, Chicago, Illinois, USA). Descriptive statistics were used to describe the basic features of the data. The Shapiro Wilk test was performed to test whether the data were normally distributed. According to the results of normality tests, repeated measures ANOVA with Bonferroni corrections (parametric test) was used to test for statistically significant differences in the measured parameters (BL concentrations, HR, BT) among different measuring points. Normally distributed data are reported as means \pm standard deviation (SD); not normally distributed data are reported as medians and interquartile range (IQR - 25th to 75th percentile). A value of $\mathrm{P}<0.05$ was considered significant.

\section{Results}

In this preliminary study we evaluated 7 dogs of the following breeds: French bulldogs $(n=6)$ and Boston terrier $(n=1)$. Baseline characteristics of the evaluated dogs are summarised in Table 1.

Table 1. Baseline characteristics of brachycephalic obstructive airway syndrome patients.

\begin{tabular}{|l|c|}
\hline Number of dogs & 7 \\
\hline Sex (female/male) & $1 / 6$ \\
\hline Age (months); Mean \pm SD & $22.14 \pm 12.41$ \\
\hline Weight (kg); Mean \pm SD & $10.91 \pm 1.23$ \\
\hline BCS Median; IQR & $3.0 ; 3.0-3.0$ \\
\hline
\end{tabular}

$\mathrm{SD}$ - standard deviation; BCS - body condition score; IQR - interquartile range (25th to 75th percentile)

Before, during and after submaximal exercise test, BL concentrations did not exceed reference ranges determined in dogs with the same portable device as in our study (Stevenson et al., 2007). Blood lactate concentrations (Figure 1) decreased significantly during the exercise testing. Compered to initial BL measurement at $\mathrm{T} 1$, significantly lower BL concentrations were observed at T3 $(\mathrm{P}=0.017$; after 10 minutes of exercise) and at $\mathrm{T} 5(\mathrm{P}=$ 0.002; after 30 minutes of resting time). The lowest BL concentration was measured at the last measuring point (after 30 minutes of recovery), when it was significantly lower compared with the initial BL concentration $(\mathrm{P}=0.002)$ and with the $\mathrm{BL}$ concentration measured at $\mathrm{T} 2(\mathrm{P}<0.001 ; 5$ minutes after the start of the exercise test).

During exercise testing, the BT values (Figure 2) changed significantly. Body temperature values were significantly higher at measuring points $\mathrm{T} 2(\mathrm{P}=0.016)$ and T3 $(\mathrm{P}=$ 0.001) compared with the initial values of BT. Afterwards, BT decreased significantly, with the last BT values being significantly lower compared to the BT values measured at T4 $(\mathrm{P}=0.018)$ and T3 $(\mathrm{P}=0.003)$ measuring points. 


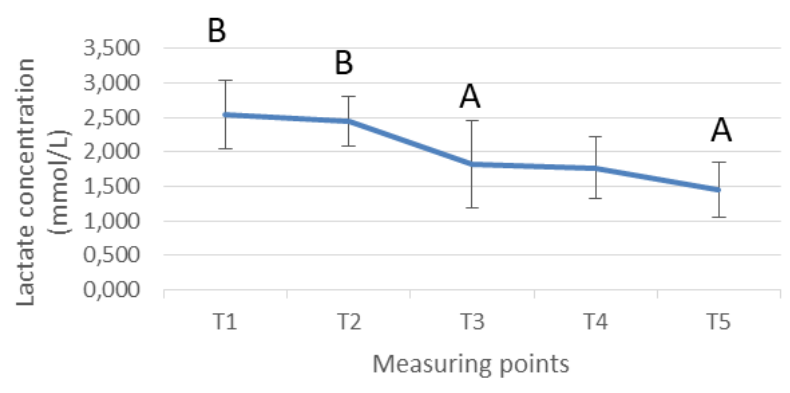

Figure 1. Blood lactate concentrations ( $\mathrm{mmol} / \mathrm{L}$; mean \pm standard deviation) before, during and after submaximal exercise test; A significant $(\mathrm{P}<0.05)$ difference compared to $\mathrm{T} 1$; $\mathrm{B}$ - significant $(\mathrm{P}<0.05)$ difference compared to T5; T1 - BL concentration before the start of ET; T2 - BL concentration after 5 min of ET; T3 - BL concentration after10 minutes of ET; T4 - BL concentration after 15 minutes of rest; T5 - BL concentration after 30 minutes of rest.

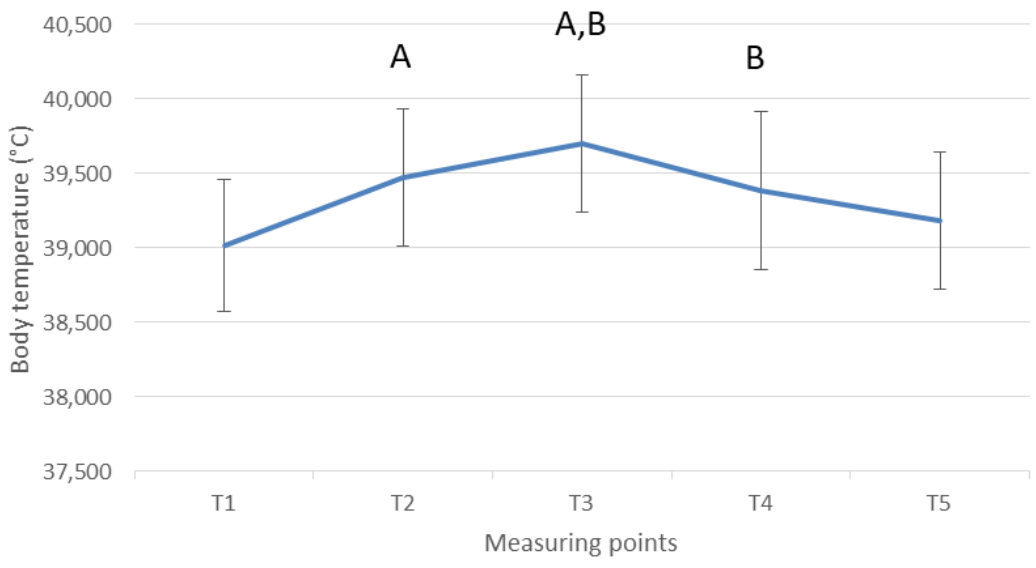

Figure 2. Body temperature $\left({ }^{\circ} \mathrm{C}\right)$ values (mean \pm standard deviation) before, during and after submaximal exercise test; A - significant $(\mathrm{P}<0.05)$ difference compared to T1; B - significant $(\mathrm{P}<0.05)$ difference compared to T5; T1 - BT before the start of ET; T2 - BT after 5 minutes of ET; T3 - BT after 10 minutes of ET; T4 - BT after 15 minutes of rest; T5 - BT after 30 minutes of rest.

The values of HR (Figure 3) showed the same pattern of changes as BT. Heart rate values were significantly higher at measuring points $T 2(\mathrm{P}=0.022)$ and $\mathrm{T} 3(\mathrm{P}=0.022)$ compared with the initial values of HR. Afterwards, HR decreased significantly with the last $\mathrm{HR}$ values being significantly lower compared with the HR values measured at $\mathrm{T} 4$ ( $\mathrm{P}$ $=0.014)$ and $\mathrm{T} 3(\mathrm{P}=0.024)$ measuring points. 


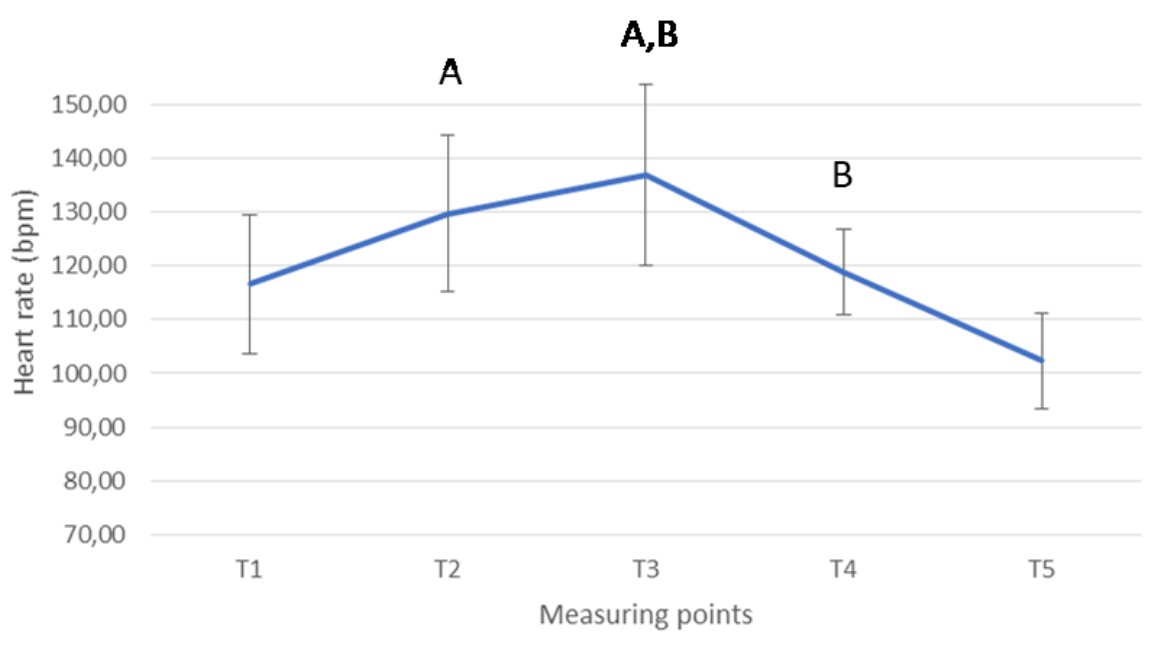

Figure 3. Heart rate values $(\mathrm{bpm})$ (mean \pm standard deviation) before, during and after submaximal exercise test; $\mathrm{A}$ - significant $(\mathrm{P}<$ 0.05) difference compared to T1; B - significant $(\mathrm{P}<0.05)$ difference compared to T5; T1 - HR before the start of ET; T2 - HR after 5 minutes of ET; T3 - HR after 10 minutes of ET; T4 - HR after 15 minutes of rest; T5 - HR after 30 minutes of rest.

\section{Discussion}

In the present study we investigated blood lactate concentrations, body temperature, and heart rate in dogs with BOAS during a submaximal exercise test. Exercise resulted in a significant increase of HR and BT and a significant decrease in BL concentrations.

To the best of the authors' knowledge there are no published reports of BL concentrations in dogs with BOAS during a submaximal exercise test. However, there are few reports (Riggs et al., 2019; Lija-Maula et al., 2017) published on exercise testing in dogs with BOAS but none of these reports was a full objective evaluation of the dogs. In the study by Riggs et al. (2019) the dogs performed a 5-minute walk and a 3-minute trot test to assess the severity of upper respiratory disease. In the study by Lija-Maula et al. (2017) they used the 6-minute walk test and the 1000 metre walk test to objectively assess the severity of BOAS in English bulldogs.

Lactate level is an indicator of physical fitness, athletic performance, and disease in animals (Ferasin and Marcora, 2007). In our study BL concentrations were highest before the start of the test. However, blood lactate concentrations decreased significantly during the test and reached the lowest BL concentration at the last measuring point. During submaximal exercise, the accumulation of lactate is reduced (Brito Vieira et al., 2014). This can be attributed to increased oxidative capacity, reduced production of lactate and an increase in the rate of lactate removal. Lactate removal may be associated with an increase in the utilisation of other tissues such as liver, heart, kidneys, and skeletal muscle. It may also be due to the increase in gluconeogenesis in skeletal muscle cells and hepatocytes, its use by oxidative fibres or increased lactate transport through the sarcolemma (Brito Vieira et al., 2014). In human athletes, lactate concentrations decreased during long endurance exercises. The authors speculated that the decrease in BL concentration was probably caused by the fact that the working muscles produced less lactate due to the lower energy demand caused by the lower respiratory demand or that the trained respiratory muscles used more lactate as fuel for their own activity (Spengler et al., 1999). The latter might be the reason for a significant decrease in BL concentration in our study. Indeed, brachycephalic dogs suffer from laboured breathing, which may result in trained respiratory muscles that use lactate as fuel.

An increase in HR and BT was expected in this study. The increase in HR is the indicator of cardiovascular workload and the most important determinant of cardiac output and oxygen uptake (Rovira et al., 2018). We observed the same pattern of significant changes in HR and BT in our BOAS patients. Both parameters increased during the exercise test and were highest after the inclination part of the test, when workload was great- 
est. Heart rate and BT began to decrease when the dogs were at rest. Despite a decrease in BT during rest, the values were still higher than BT before the start of the workload. This is due to the increased muscle load, which generates higher amounts of body heat (Rovira et al., 2018).

Our preliminary results warrant further studies with a larger number of BOAS patients to evaluate the physiological responses to submaximal exercise testing in individual brachycephalic breeds and to determine if surgical treatment of BOAS affects responses to this type of exercise.

\section{Conclusions}

The present study demonstrated that submaximal exercise of only 10 minutes duration caused a significant increase in HR and BT, and surprisingly, a significant decrease in BL concentrations. The results of this preliminary study may give us additional information about the severity of BOAS. However, further studies are needed to gain better insight into the physiological response of BOAS patients of individual breeds to submaximal exercise testing.

Funding: This research was supported by Slovenian Research Agency (research program No. P4-0053).

Institutional Review Board Statement: All procedures complied with the relevant Slovenian governmental regulations (Animal Protection Act UL RS, 43/2007).

Conflicts of Interest: The authors declare no conflict of interest.

Acknowledgements: The authors thank Janez Vodičar from Laboratory of Physiology, Faculty of Sports, University of Ljubljana, for providing the facilities and for help with the use of the equipment.

\section{References}

1. Alves JC, Santos AM, Jorge PI, Lafuente P. A protocol for the determination of the maximal lactate steady state in working dogs. J Sports Med Phys Fitness. 2020; 60: 942-946. DOI: 10.23736/S0022-4707.20.10305-0.

2. Brito Vieira WH, Halsberghe MJ, Schwantes ML et al. Increased lactate threshold after five weeks of treadmill aerobic training in rats. Braz J Biol. 2014; 74: 444-9. DOI: 10.1590/1519-6984.07912.

3. Brooks GA. The Science and Translation of Lactate Shuttle Theory. Cell Metab. 2018; 27(4):757-785. DOI: 10.1016/j.cmet.2018.03.008.

4. Dupré G, Heidenreich D. Brachycephalic Syndrome. Vet Clin North Am Small Anim Pract. 2016; 46, 691-707. DOI: 10.1016/j.cvsm.2016.02.002.

5. Ekenstedt KJ, Crosse KR, Risselada M. Canine Brachycephaly: Anatomy, Pathology, Genetics and Welfare. J Comp Pathol. 2020; 176: 109-115. DOI: 10.1016/j.jcpa.2020.02.008.

6. Erjavec V, Vovk T, Svete AN. Evaluation of Oxidative Stress Parameters in Dogs with Brachycephalic Obstructive Airway Syndrome Before and after Surgery. J Vet Res. 2021; 65(2):201-208. DOI: 10.2478/jvetres-2021-0027.

7. Ferasin L, Marcora S. A pilot study to assess the feasibility of a submaximal exercise test to measure individual response to cardiac medication in dogs with acquired heart failure. Vet Res Commun. 2007; 31: 725-37. DOI: 10.1007/s11259-007-3566-7.

8. Lilja-Maula L, Lappalainen AK, Hyytiäinen HK et al. Comparison of submaximal exercise test results and severity of brachycephalic obstructive airway syndrome in English bulldogs. Vet J. 2017; 219: 22-26. DOI: 10.1016/j.tvj1.2016.11.019

9. Lodato DL, Hedlund CS. Brachycephalic airway syndrome: pathophysiology and diagnosis. Compend Contin Educ Vet. 2012; 34: E3.

10. Meola SD. Brachycephalic airway syndrome. Top Companion Anim Med. 2013; 28: 91-6. DOI: 10.1053/j.tcam.2013.06.004.

11. Riggs J, Liu NC, Sutton DR, et al. Validation of exercise testing and laryngeal auscultation for grading brachycephalic obstructive airway syndrome in pugs, French bulldogs, and English bulldogs by using whole-body barometric plethysmography. Vet Surg. 2019; 48: 488-496. DOI: 10.1111/vsu.13159.

12. Environmental and Physiological Factors Associated With Stamina in Dogs Exercising in High Ambient Temperatures. Front Vet Sci. 2017; 4: 144. DOI: 10.3389/fvets.2017.00144.

13. Rovira S, Munoz A, Benito M. Effect of exercise on physiological, blood and endocrine parameters in search and rescue-trained dogs. Vet Med. 2008; 53: 333-346. DOI:10.17221/1860-VETMED

14. Spengler CM, Roos M, Laube SM, Boutellier U. Decreased exercise blood lactate concentrations after respiratory endurance training in humans. Eur J Appl Physiol Occup Physiol. 1999; 79: 299-305. DOI: 10.1007/s004210050511.

15. Stevenson CK, Kidney BA, Duke T, et al. Evaluation of the Accutrend for lactate measurement in dogs. Vet Clin Pathol. 2007; 36: 261-266. DOI: 10.1111/j.1939-165x.2007.tb00221.x 